\title{
Reconstructing an Even Damping from a Single Spectrum
}

\author{
Steven J. Cox and Mark Embree \\ Department of Computational and Applied Mathematics, Rice University, \\ 6100 Main Street - MS 134, Houston, Texas, 77005-1892, USA \\ E-mail: cox@rice.edu, embree@rice.edu
}

\begin{abstract}
We consider the wave equation on a finite interval with fixed ends and nonuniform viscous damping. We prove that the spectrum of the associated damped wave operator uniquely determines an even damping. We then develop a refined asymptotic formula for the high frequencies. When the damping is even about the domain's midpoint, terms in this expansion are Fourier coefficients for functions of the damping. Furthermore, the expansion is often quite accurate even at low frequencies, thus suggesting a simple numerical procedure for reconstructing even damping coefficients from measured eigenvalues. Computational examples illustrate the efficacy of this procedure, even in the presence of noise.
\end{abstract}

AMS classification scheme numbers: 35P20,35R30

\section{Introduction}

The displacement $u$ of a string of unit length, fixed at its ends, and in the presence of viscous damping $2 a$, solves the boundary value problem

$$
\begin{gathered}
u_{t t}(x, t)-u_{x x}(x, t)+2 a(x) u_{t}(x, t)=0, \quad 0<x<1,0<t, \\
u(0, t)=u(1, t)=0, \quad 0<t,
\end{gathered}
$$

upon being set in motion by the initial disturbance $\left[u(x, 0), u_{t}(x, 0)\right] \in H_{0}^{1}(0,1) \times$ $L^{2}(0,1)$. We equip this space with the inner product

$$
\langle[f, g],[u, v]\rangle=\int_{0}^{1} f^{\prime} \bar{u}^{\prime}+g \bar{v} \mathrm{~d} x
$$

and interpret (1) as the system $V_{t}=A(a) V$, where $V=\left[u, u_{t}\right]$ and

$$
A(a)=\left[\begin{array}{cc}
0 & I \\
\mathrm{~d}^{2} / \mathrm{d} x^{2} & -2 a
\end{array}\right], \quad \operatorname{Dom}(A)=\left(H^{2}(0,1) \cap H_{0}^{1}(0,1)\right) \times H_{0}^{1}(0,1) .
$$

If $a \in L^{\infty}(0,1)$ then $A(a)$ has a compact inverse and so a discrete spectrum, denoted $\sigma(A(a))$. In Section 2 we prove that no two even Lipschitz dampings may have the same spectrum. In Section 3 we derive refined asymptotic formulas for the large eigenvalues and argue in Section 4 that they permit us to reconstruct the unknown damping. In Section 5 we examine the performance of this approach through a variety of numerical examples.

Our uniqueness argument follows from the work of Yamamoto [1] and Cox and Knobel [2]. Our asymptotic work is a refinement of Cox and Zuazua [3]. 
Reconstructing an Even Damping from a Single Spectrum

\section{Uniqueness}

We establish uniqueness in the more general context of Yamamoto [1]. In particular, consider the operator

$$
Y(a)\left[U_{1}, U_{2}\right] \equiv\left[-U_{2}^{\prime},-U_{1}^{\prime}-2 a U_{2}\right]
$$

acting on $\operatorname{Dom}(Y)=H^{1}(0,1) \times H_{0}^{1}(0,1)$. It was proven in [2] that this operator has a discrete spectrum and that each eigenvalue, $\lambda$, is geometrically simple (the dimension of the null space of $Y(a)-\lambda$ is one) and algebraically finite (there exists a smallest positive integer $m$ for which $\left.\operatorname{Ker}(Y(a)-\lambda)^{m}=\operatorname{Ker}(Y(a)-\lambda)^{m+1}\right)$. If $U \equiv U^{0}$ is an eigenvector, then construct a basis of generalized eigenvectors via

$$
(Y(a)-\lambda) U^{j}=U^{j-1}, \quad\left\langle U^{j}, U^{0}\right\rangle_{L^{2} \times L^{2}}=0, \quad j=1, \ldots, m-1 .
$$

We first confirm that the spectra of our two operators (largely) coincide.

Theorem $1 \sigma(Y(a))=\sigma(A(a)) \cup\{0\}$ including multiplicities.

Proof. If $A(a) V=\lambda V$, then $V_{2}=\lambda V_{1}$ and $V_{1}^{\prime \prime}-2 a V_{2}=\lambda V_{2}$. On setting

$$
U_{1}=-V_{1}^{\prime} \text { and } U_{2}=V_{2}
$$

we find $Y(a) U=\lambda U$, while $V_{1} \in H_{0}^{1}(0,1)$ and $U_{2}=V_{2}=\lambda V_{1}$ implies $U_{2} \in H_{0}^{1}(0,1)$. The same transform takes generalized eigenvectors of $A(a)$ to generalized eigenvectors of $Y(a)$. In particular, if $(A(a)-\lambda) V^{1}=V$ with $U_{1}^{1}=-\partial_{x} V_{1}^{1}$ and $U_{2}^{1}=V_{2}^{1}$, then $(Y(a)-\lambda) U^{1}=U$.

Conversely, if $Y(a) U=\lambda U$ then

$$
V_{1}=-\int_{0}^{x} U_{1}(y) \mathrm{d} y \quad \text { and } \quad V_{2}=U_{2}
$$

satisfies $A(a) V=\lambda V$, while $-U_{2}^{\prime}=\lambda U_{1}$ and $U_{2} \in H_{0}^{1}(0,1)$ implies

$$
\lambda \int_{0}^{1} U_{1}(y) \mathrm{d} y=0 .
$$

It follows that if $\lambda \neq 0$ then $V_{1} \in H_{0}^{1}(0,1)$. This same transform takes generalized eigenvectors of $Y(a)$ to those of $A(a)$. It follows that the nonzero eigenvalues of $Y(a)$ coincide with those of $A(a)$. The zero eigenvalue of $Y(a)$ is algebraically simple and its root space is spanned by $[1,0]$.

We will show that $\sigma(Y(a))$ uniquely determines an even $a$ by extending the classic argument, e.g., see Gantmacher and Krein [4], that in such a case (i) $\sigma(Y(a))$ actually encodes two spectra of $a$ on the half-string $[0,1 / 2]$ and (ii) that these two spectra uniquely determine $a$.

The two relevant spectra come from the restriction of $Y(a)$ to the domains

$$
\begin{aligned}
& \operatorname{Dom}_{D, D_{1 / 2}}=\left\{U \in H^{1}(0,1 / 2)^{2}: U_{2}(0)=U_{2}(1 / 2)=0\right\} \\
& \operatorname{Dom}_{D, N_{1 / 2}}=\left\{U \in H^{1}(0,1 / 2)^{2}: U_{2}(0)=U_{1}(1 / 2)=0\right\} .
\end{aligned}
$$

We denote the spectra of these restrictions by $\sigma_{D, D_{1 / 2}}(a)$ and $\sigma_{D, N_{1 / 2}}(a)$, and denote by $\sigma_{D, D_{1}}(a) \equiv \sigma(Y(a))$ the associated $(D, D)$ spectrum on the full string. Corollary 1.2 of [2] establishes the following result.

Theorem 2 If $a, b \in \operatorname{Lip}(0,1 / 2)$ satisfy $\sigma_{D, D_{1 / 2}}(a)=\sigma_{D, D_{1 / 2}}(b)$ and $\sigma_{D, N_{1 / 2}}(a)=$ $\sigma_{D, N_{1 / 2}}(b)$, then $a=b$. 
It remains only to equate the spectrum on the full string with the union of the two half-string spectra when $a$ is even about the midpoint of the domain $[0,1]$.

Theorem 3 If $a \in \operatorname{Lip}(0,1)$ is even, then $\sigma_{D, D_{1}}(a)=\sigma_{D, D_{1 / 2}}(a) \cup \sigma_{D, N_{1 / 2}}(a)$.

Proof. We show that $\sigma_{D, D_{1 / 2}}(a) \cup \sigma_{D, N_{1 / 2}}(a) \subset \sigma_{D, D_{1}}(a)$ by odd extension of the component that vanishes at $1 / 2$ and even extension of the other component. We begin with a $\left(D, D_{1 / 2}\right)$ eigenpair $(\mu, \Phi)$ and construct the $\left(D, D_{1}\right)$ eigenfunction

$U_{1}(x)=\left\{\begin{array}{ll}\Phi_{1}(x), & x \in(0,1 / 2) ; \\ \Phi_{1}(1-x), & x \in(1 / 2,1) ;\end{array} \quad\right.$ and $\quad U_{2}(x)= \begin{cases}\Phi_{2}(x), & x \in(0,1 / 2) ; \\ -\Phi_{2}(1-x), & x \in(1 / 2,1),\end{cases}$

associated with $\mu$. If $\mu$ is a double $\left(D, D_{1 / 2}\right)$ eigenvalue then there exists a generalized eigenvector $\Phi^{1}$ such that

$$
(Y(a)-\mu) \Phi^{1}=\Phi, \quad \Phi_{2}^{1}(0)=\Phi_{2}^{1}(1 / 2)=0 .
$$

If $U_{1}^{1}$ is the even extension of $\Phi_{1}^{1}$ and $U_{2}^{1}$ is the odd extension of $\Phi_{2}^{1}$, then $U^{1}$ indeed obeys

$$
(Y(a)-\mu) U^{1}=U, \quad U_{2}^{1}(0)=U_{2}^{1}(1)=0 .
$$

This procedure generalizes immediately to higher multiplicities. We have now shown that $\sigma_{D, D_{1 / 2}}(a) \subset \sigma_{D, D_{1}}(a)$.

Similarly, from a $\left(D, N_{1 / 2}\right)$ eigenpair $(\nu, \Psi)$ we construct the $\left(D, D_{1}\right)$ eigenfunction

$U_{1}(x)=\left\{\begin{array}{ll}\Psi(x), & x \in(0,1 / 2) ; \\ -\Psi_{1}(1-x), & x \in(1 / 2,1) ;\end{array} \quad\right.$ and $\quad U_{2}(x)= \begin{cases}\Psi_{2}(x), & x \in(0,1 / 2) ; \\ \Psi_{2}(1-x), & x \in(1 / 2,1),\end{cases}$

associated with $\nu$. As above, if $\Psi^{k}$ is an associated generalized $\left(D, N_{1 / 2}\right)$ eigenvector and $U_{1}^{k}$ is the odd extension of $\Psi_{1}^{k}$ and $U_{2}^{k}$ is the even extension of $\Psi_{2}^{k}$, then $U^{k}$ is an associated generalized $\left(D, D_{1}\right)$ eigenvector and so $\sigma_{D, N_{1 / 2}}(a) \subset \sigma_{D, D_{1}}(a)$. We have now shown that $\sigma_{D, D_{1 / 2}}(a) \cup \sigma_{D, N_{1 / 2}}(a) \subset \sigma_{D, D_{1}}(a)$.

Conversely, if $(U, \lambda)$ is a $\left(D, D_{1}\right)$ eigenpair, then $\left(\left[-U_{1}(1-x), U_{2}(1-x)\right], \lambda\right)$ is also a $\left(D, D_{1}\right)$ eigenpair, since $a$ is even. As the geometric multiplicity of $\lambda$ is one, it follows that $U_{1}(x)=-c U_{1}(1-x)$ and $U_{2}(x)=c U_{2}(1-x)$ for some scalar $c$. Note that if $U_{1}$ and $U_{2}$ vanish at the same point, then both must vanish in the entire interval.

If $U_{1}(1 / 2) \neq 0$ then $c=-1$ and $U_{2}(1 / 2)=-U_{2}(1 / 2)=0$ and so $(U, \lambda)$ is a $\left(D, D_{1 / 2}\right)$ eigenpair. Similarly, if $U_{2}(1 / 2) \neq 0$ then $c=1$ and $U_{1}(1 / 2)=-U_{1}(1 / 2)=0$ and so $(U, \lambda)$ is a $\left(D, N_{1 / 2}\right)$ eigenpair.

If $\left(U^{1}, \lambda\right)$ is a generalized $\left(D, D_{1}\right)$ eigenvector, i.e.,

$$
(Y(a)-\lambda) U^{1}=U, \quad U_{2}^{1}(0)=U_{2}^{1}(1)=0,
$$

then $U^{1}$ inherits the symmetries of $U$. In particular, if $U_{1}$ is even and $U_{2}$ is odd, then $U_{1}^{1}$ is even and $U_{2}^{1}$ is odd. It follows that $\sigma_{D, D_{1}}(a) \subset \sigma_{D, D_{1 / 2}}(a) \cup \sigma_{D, N_{1 / 2}}(a)$.

Corollary 1 If $a \in \operatorname{Lip}(0,1)$ is even, then $\sigma_{D, D_{1}}(a)$ uniquely determines a. 


\section{Asymptotic Expansion}

We begin with the expansion of Cox and Zuazua, in terms of

$$
a(x)=a_{0}+\widetilde{a}(x), \quad a_{0} \equiv \int_{0}^{1} a(x) \mathrm{d} x \quad \text { and } \quad \xi(x) \equiv \int_{0}^{x} \widetilde{a}(r) \mathrm{d} r .
$$

Theorem 4 [3, Theorem 5.5] If $a \in B V(0,1)$ and $A(a) V_{n}=\lambda_{n} V_{n}$ then $V_{n}(x)=$ $g_{n}(x)\left[\begin{array}{ll}1 & \lambda_{n}\end{array}\right]$, where

$$
\begin{aligned}
& \lambda_{n}(a)=-a_{0}+i n \pi+O(1 /|n|) \\
& g_{n}(x ; a)=\frac{\sinh (\xi(x)+i n \pi x)}{-a_{0}+i n \pi}+O\left(1 / n^{2}\right) \\
& g_{n}^{\prime}(x ; a)=\cosh (\xi(x)+i n \pi x)+O(1 /|n|) .
\end{aligned}
$$

Our principal goal is to specify the $O(1 /|n|)$ term in the expression for $\lambda_{n}$. We will accomplish this, following Pöschel and Trubowitz [5, Theorem 2.4], by exploiting the simple identity

$$
\lambda_{n}\left(a_{0}+\widetilde{a}\right)-\lambda_{n}\left(a_{0}\right)=\int_{0}^{1} \frac{\mathrm{d}}{\mathrm{d} t} \lambda_{n}\left(a_{0}+t \widetilde{a}\right) \mathrm{d} t .
$$

If the mean $a_{0}$ is not an integer multiple of $\pi$ and the deviation $\widetilde{a}$ is small, then the derivative in the integrand may be evaluated through formal differentiation of

$$
A\left(a_{0}+t \widetilde{a}\right) V_{n}\left(a_{0}+t \widetilde{a}\right)=\lambda_{n}\left(a_{0}+t \widetilde{a}\right) V_{n}\left(a_{0}+t \widetilde{a}\right) .
$$

Hence, with an over-dot denoting $\mathrm{d} / \mathrm{d} t$,

$$
\begin{aligned}
\dot{A}\left(a_{0}+t \widetilde{a}\right) V_{n}\left(a_{0}+t \widetilde{a}\right) & +A\left(a_{0}+t \widetilde{a}\right) \dot{V}_{n}\left(a_{0}+t \widetilde{a}\right) \\
& =\dot{\lambda}_{n}\left(a_{0}+t \widetilde{a}\right) V_{n}\left(a_{0}+t \widetilde{a}\right)+\lambda_{n}\left(a_{0}+t \widetilde{a}\right) \dot{V}_{n}\left(a_{0}+t \widetilde{a}\right) .
\end{aligned}
$$

To isolate the desired term we take the inner product, defined in (2), of each side with the associated adjoint eigenvector, $W_{n}$. For, as $W_{n}$ obeys

$$
A^{*}\left(a_{0}+t \widetilde{a}\right) W_{n}\left(a_{0}+t \widetilde{a}\right)=\overline{\lambda_{n}}\left(a_{0}+t \widetilde{a}\right) W_{n}\left(a_{0}+t \widetilde{a}\right)
$$

we obtain, on abbreviating each argument, $a_{0}+t \widetilde{a}$, as simply $t$,

$$
\left\langle\dot{A}(t) V_{n}(t), W_{n}(t)\right\rangle=\dot{\lambda}_{n}(t)\left\langle V_{n}(t), W_{n}(t)\right\rangle .
$$

Regarding the right side, we recall [3, p. 217] that

$$
W_{n}(t)=\overline{g_{n}}(x ; t)\left[1,-\overline{\lambda_{n}}(t)\right]
$$

and find

$$
\begin{aligned}
\left\langle V_{n}(t), W_{n}(t)\right\rangle & =\int_{0}^{1}\left(g_{n}^{\prime}(x ; t)\right)^{2}-\lambda_{n}^{2}(t) g_{n}^{2}(x ; t) \mathrm{d} x \\
& =\int_{0}^{1}\left\{\cosh ^{2}(t \xi(x)+i n \pi x)-\sinh ^{2}(t \xi(x)+i n \pi x)\right\} \mathrm{d} x+O(1 /|n|) \\
& =1+O(1 /|n|) .
\end{aligned}
$$

We next move to the left side of (5), record

$$
\dot{A}(t) V_{n}(t)=\left[0,-2 \widetilde{a}(x) \lambda_{n}(t) g_{n}(x ; t)\right]
$$


and proceed to evaluate

$$
\begin{aligned}
\left\langle\dot{A}(t) V_{n}(t), W_{n}(t)\right\rangle & =\int_{0}^{1}\left(-2 \widetilde{a}(x) \lambda_{n}(t) g_{n}(x ; t) \overline{\left(-\overline{g_{n}(x ; t)} \overline{\lambda_{n}(t)}\right)} \mathrm{d} x\right. \\
& =2 \lambda_{n}^{2}(t) \int_{0}^{1} \widetilde{a}(x) g_{n}^{2}(x ; t) \mathrm{d} x \\
& =2 \int_{0}^{1} \widetilde{a}(x) \sinh ^{2}(t \xi(x)+i n \pi x) \mathrm{d} x+O(1 /|n|) .
\end{aligned}
$$

Our precise task is to reveal this latter $O(1 /|n|)$ term. To do this requires that we extend the estimate from [3] of the associated shooting function. To be precise, recall that if $V=[y, z]$ is an eigenvector of $A$ with eigenvalue $\lambda$, then $z=\lambda y$ and $y^{\prime \prime}-2 a z=\lambda z$, and so $y \in H_{0}^{1}(0,1)$ must satisfy

$$
y^{\prime \prime}-\lambda^{2} y-2 a \lambda y=0 .
$$

We denote by $y_{2}(x, \lambda)$ the solution of $(8)$ subject to the initial conditions

$$
y(0, \lambda)=0, \quad y^{\prime}(0, \lambda)=1 .
$$

The eigenvalues of $A(a)$ are then the zeros of $\lambda \mapsto y_{2}(1, \lambda)$. On following a classical ansatz, Cox and Zuazua [3] argue that $y_{2}$ is close to the exact solution

$z_{2}(x, \lambda)=\frac{1}{\lambda} \sinh (p(x))+\frac{1}{\lambda} \mathrm{e}^{p(x)} \int_{0}^{x} a(r) \mathrm{e}^{-2 p(r)} \mathrm{d} r, \quad p(x) \equiv \lambda x+\int_{0}^{x} a(r) \mathrm{d} r$

of the associated initial value problem

$$
-z^{\prime \prime}+\lambda^{2} z+2 \lambda a z+\left(a^{2}+a^{\prime}\right) z=0, \quad z(0, \lambda)=0, z^{\prime}(0, \lambda)=1 .
$$

Integration by parts isolates the $1 / \lambda$ term in $z_{2}$,

$z_{2}(x, \lambda)=\frac{1}{\lambda} \sinh (p(x))-\frac{\mathrm{e}^{p(x)}}{2 \lambda^{2}}\left(a(0)-a(x) \mathrm{e}^{-2 p(x)}+\int_{0}^{x}\left(a^{\prime}(r)-2 a^{2}(r)\right) \mathrm{e}^{-2 p(r)} \mathrm{d} r\right)$,

and, provided $a \in H^{2}(0,1)$, we may integrate by parts again to reveal the $1 / \lambda^{2}$ term,

$$
z_{2}(x, \lambda)=\frac{\sinh (p(x))}{\lambda}-\frac{a(0) \mathrm{e}^{p(x)}-a(x) \mathrm{e}^{-p(x)}}{2 \lambda^{2}}+O\left(1 / \lambda^{3}\right) .
$$

From [3, Eq. (5.6)], we recall that $y_{2}$ is close to $z_{2}$ in the sense that

$$
y_{2}(x, \lambda)=z_{2}(x, \lambda)+S_{1}(x, \lambda)+O\left(1 / \lambda^{3}\right),
$$

where

$$
S_{1}(x, \lambda)=\int_{0}^{x} K(x, r, \lambda)\left(a^{2}(r)+a^{\prime}(r)\right) z_{2}(r, \lambda) \mathrm{d} r
$$

and the kernel

$$
\begin{aligned}
K(x, r, \lambda) & =-\frac{1}{\lambda} \sinh (p(x)-p(r))+\frac{1}{\lambda} \mathrm{e}^{p(x)+p(r)} \int_{r}^{x} a(s) \mathrm{e}^{-2 p(s)} \mathrm{d} s \\
& =-\frac{1}{\lambda} \sinh (p(x)-p(r))+O\left(1 / \lambda^{2}\right) .
\end{aligned}
$$


We proceed to evaluate $S_{1}$ up to $O\left(1 / \lambda^{3}\right)$. To do this, we need only retain $z_{2}$ and $K$ up to $O(1 / \lambda)$, i.e.,

$$
\begin{aligned}
S_{1}(x, \lambda)= & -\frac{1}{\lambda^{2}} \int_{0}^{x} \sinh (p(x)-p(r))\left(a^{2}(r)+a^{\prime}(r)\right) \sinh (p(r)) \mathrm{d} r+O\left(1 / \lambda^{3}\right) \\
= & -\frac{1}{2 \lambda^{2}} \int_{0}^{x}\{\cosh (p(x))-\cosh (p(x)-2 p(r))\}\left(a^{2}(r)+a^{\prime}(r)\right) \mathrm{d} r+O\left(1 / \lambda^{3}\right) \\
= & -\frac{1}{2 \lambda^{2}} \cosh (p(x))\left(a(x)-a(0)+\int_{0}^{x} a^{2}(r) \mathrm{d} r\right) \\
& +\frac{1}{2 \lambda^{2}} \int_{0}^{x} \cosh (p(x)-2 p(r))\left(a^{2}(r)+a^{\prime}(r)\right) \mathrm{d} r+O\left(1 / \lambda^{3}\right) \\
= & \frac{1}{2 \lambda^{2}} \cosh (p(x))\left(a(x)-a(0)+\int_{0}^{x} a^{2}(r) \mathrm{d} r\right)+O\left(1 / \lambda^{3}\right),
\end{aligned}
$$

where the last line follows from yet another integration by parts, under the assumption that $a \in H^{2}(0,1)$. Finally, substituting this expression for $S_{1}$ into (11) yields a formula for $y_{2}$ that captures both the $1 / \lambda$ and $1 / \lambda^{2}$ terms:

$$
\begin{aligned}
y_{2}(x, \lambda)= & \frac{\sinh (p(x))}{\lambda}-\frac{(a(0)+a(x)) \sinh (p(x))+\cosh (p(x)) \int_{0}^{x} a^{2}(r) \mathrm{d} r}{2 \lambda^{2}} \\
& +O\left(1 / \lambda^{3}\right) .
\end{aligned}
$$

To develop an expansion for (5), we begin by noting that key terms in (7) can be estimated using Theorem 4 and (12):

$$
\begin{aligned}
\lambda_{n}(t) g_{n}(x ; t)=\lambda_{n}(t) y_{2}\left(x, \lambda_{n}(t)\right) \\
=\sinh \left(\lambda_{n}(t) x+\int_{0}^{x}\left(a_{0}+t \widetilde{a}(r)\right) \mathrm{d} r\right) \\
\quad-\frac{1}{2 \lambda_{n}(t)}\left(a_{0}+t \widetilde{a}(0)+a_{0}+t \widetilde{a}(x)\right) \sinh \left(\lambda_{n}(t) x+\int_{0}^{x}\left(a_{0}+t \widetilde{a}(r)\right) \mathrm{d} r\right) \\
\quad-\frac{1}{2 \lambda_{n}(t)} \cosh \left(\lambda_{n}(t) x+\int_{0}^{x}\left(a_{0}+t \widetilde{a}(r)\right) \mathrm{d} r\right) \int_{0}^{x}\left(a_{0}+t \widetilde{a}(r)\right)^{2} \mathrm{~d} r+O\left(1 / n^{2}\right) .
\end{aligned}
$$

On squaring we find

$$
\begin{aligned}
\lambda_{n}^{2}(t) g_{n}^{2}(x ; t)= & \sinh ^{2}(t \xi(x)+i n \pi x+O(1 /|n|)) \\
& -\frac{1}{\lambda_{n}(t)}\left(2 a_{0}+t(\widetilde{a}(0)+\widetilde{a}(x))\right) \sinh ^{2}(t \xi(x)+i n \pi x+O(1 /|n|)) \\
& -\frac{1}{\lambda_{n}(t)} \sinh (2 t \xi(x)+i 2 n \pi x+O(1 /|n|)) \int_{0}^{x}\left(a_{0}+t \widetilde{a}(r)\right)^{2} \mathrm{~d} r .
\end{aligned}
$$

On substituting this result into (7) we find

$$
\left\langle\dot{A}(t) V_{n}(t), W_{n}(t)\right\rangle=2 \lambda_{n}^{2}(t) \int_{0}^{1} \widetilde{a}(x) g_{n}^{2}(x ; t) \mathrm{d} x=I_{1}(t)-I_{2}(t)-I_{3}(t),
$$

where

$$
\begin{aligned}
I_{1}(t) & =2 \int_{0}^{1} \widetilde{a}(x) \sinh ^{2}(t \xi(x)+i n \pi x+O(1 /|n|)) \mathrm{d} x \\
& =\int_{0}^{1} \widetilde{a}(x) \cosh (2 t \xi(x)+i 2 n \pi x+O(1 /|n|)) \mathrm{d} x
\end{aligned}
$$


Reconstructing an Even Damping from a Single Spectrum

$$
\begin{aligned}
& =\int_{0}^{1} \widetilde{a}(x)(\cosh (2 t \xi(x)+i 2 n \pi x)+O(1 /|n|) \sinh (2 t \xi(x)+i 2 n \pi x)) \mathrm{d} x \\
& =\int_{0}^{1} \widetilde{a}(x)(\cosh (2 t \xi(x)) \cos (2 n \pi x)+i \sinh (2 t \xi(x)) \sin (2 n \pi x)) \mathrm{d} x+O\left(1 / n^{2}\right), \\
I_{2}(t) & =\frac{2}{\lambda_{n}(t)} \int_{0}^{1} \widetilde{a}(x)\left(2 a_{0}+t(\widetilde{a}(0)+\widetilde{a}(x))\right) \sinh ^{2}(t \xi(x)+i n \pi x+O(1 /|n|)) \mathrm{d} x \\
& =\frac{1}{\lambda_{n}(t)} \int_{0}^{1} \widetilde{a}(x)\left(2 a_{0}+t(\widetilde{a}(0)+\widetilde{a}(x))\right) \cosh (2 t \xi(x)+i 2 n \pi x+O(1 /|n|)) \mathrm{d} x \\
& \quad-\frac{t}{\lambda_{n}(t)} \int_{0}^{1} \widetilde{a}^{2}(x) \mathrm{d} x \\
= & \frac{i t}{n \pi} \int_{0}^{1} \widetilde{a}^{2}(x) \mathrm{d} x+O\left(1 / n^{2}\right),
\end{aligned}
$$

and finally

$$
\begin{aligned}
I_{3}(t) & =\frac{1}{\lambda_{n}(t)} \int_{0}^{1} \widetilde{a}(x)\left(\int_{0}^{x}\left(a_{0}+t \widetilde{a}(r)\right)^{2} \mathrm{~d} r\right) \sinh (2 t \xi(x)+i 2 n \pi x+O(1 /|n|)) \mathrm{d} x \\
& =O\left(1 / n^{2}\right) .
\end{aligned}
$$

Having compiled each of the terms in (4), we now evaluate

$$
\begin{aligned}
\int_{0}^{1} \dot{\lambda}\left(a_{0}+t \widetilde{a}\right) \mathrm{d} t= & \int_{0}^{1} \frac{\left\langle\dot{A}\left(a_{0}+t \widetilde{a}\right) V_{n}\left(a_{0}+t \widetilde{a}\right), W_{n}\left(a_{0}+t \widetilde{a}\right)\right\rangle}{\left\langle V_{n}\left(a_{0}+t \widetilde{a}\right), W_{n}\left(a_{0}+t \widetilde{a}\right)\right\rangle} \mathrm{d} t \\
= & \int_{0}^{1} \frac{I_{1}(t)+I_{2}(t)+I_{3}(t)}{1+O(1 /|n|)} \mathrm{d} t \\
= & \int_{0}^{1} \widetilde{a}(x) \frac{\sinh (2 \xi(x))}{2 \xi(x)} \cos (2 n \pi x) \mathrm{d} x \\
& +i \int_{0}^{1} \widetilde{a}(x) \frac{\cosh (2 \xi(x))-1}{2 \xi(x)} \sin (2 n \pi x) \mathrm{d} x \\
& \quad-\frac{i}{2 n \pi} \int_{0}^{1} \widetilde{a}^{2}(x) \mathrm{d} x+O\left(1 / n^{2}\right) .
\end{aligned}
$$

Substituting this expansion into (4), together with

$$
\lambda_{n}\left(a_{0}\right)=-a_{0}+\sqrt{a_{0}^{2}-n^{2} \pi^{2}}=-a_{0}+i\left(n \pi-\frac{a_{0}^{2}}{2 n \pi}\right)+O\left(1 / n^{3}\right),
$$

gives following refinement of Theorem 4 .

Theorem 5 If $a \in H^{2}(0,1)$ then $\lambda_{n}(a)=\widetilde{\lambda}_{n}(a)+O\left(1 / n^{2}\right)$ where

$$
\begin{aligned}
\widetilde{\lambda}_{n}(a) \equiv & -a_{0}+\int_{0}^{1} \widetilde{a}(x) \frac{\sinh (2 \xi(x))}{2 \xi(x)} \cos (2 n \pi x) \mathrm{d} x \\
& +i n \pi-\frac{i}{2 n \pi} \int_{0}^{1} a^{2}(x) \mathrm{d} x+i \int_{0}^{1} \widetilde{a}(x) \frac{\cosh (2 \xi(x))-1}{2 \xi(x)} \sin (2 n \pi x) \mathrm{d} x,
\end{aligned}
$$

and $a_{0}, \widetilde{a}$ and $\xi$ remain as defined in (3). 
Reconstructing an Even Damping from a Single Spectrum

Table 1. Low-frequency eigenvalues (to five places, via a high-accuracy spectral discretization) for the damping function $a(x)=1-\sin (11 \pi x) \exp \left(-10(x-1 / 2)^{2}\right)$, along with the error $\left|\lambda_{n}(a)-\widetilde{\lambda}_{n}(a)\right|$ in the approximation from Theorem 5. (All quantities are believed to be correct to the digits provided.)

\begin{tabular}{rcc}
\hline$n$ & $\lambda_{n}(a)$ & error \\
\hline 1 & $-1.00122+2.97964 i$ & 0.0269879 \\
2 & $-1.00106+6.20514 i$ & 0.0167440 \\
3 & $-1.00228+9.37615 i$ & 0.0144677 \\
4 & $-0.97180+12.53868 i$ & 0.0197670 \\
5 & $-1.21888+15.66851 i$ & 0.0022341 \\
6 & $-0.77494+18.82612 i$ & 0.0080114 \\
7 & $-1.02197+21.95436 i$ & 0.0097917 \\
8 & $-0.99174+25.10548 i$ & 0.0036935 \\
9 & $-0.99287+28.25021 i$ & 0.0026511 \\
10 & $-0.99374+31.39954 i$ & 0.0005894
\end{tabular}

We note that the fourth term in (13) was recently discovered by Borisov and Freitas [6]. Remarkably, we often find the expansion in Theorem 5 to be quite accurate even for small values of $n$. To investigate, we require access to the 'exact' eigenvalues $\lambda_{n}(a)$. High-accuracy approximations follow from a Chebyshev pseudospectral collocation discretization of $A(a)$, requiring only a few lines of MATLAB code and Trefethen's cheb.m routine [7].

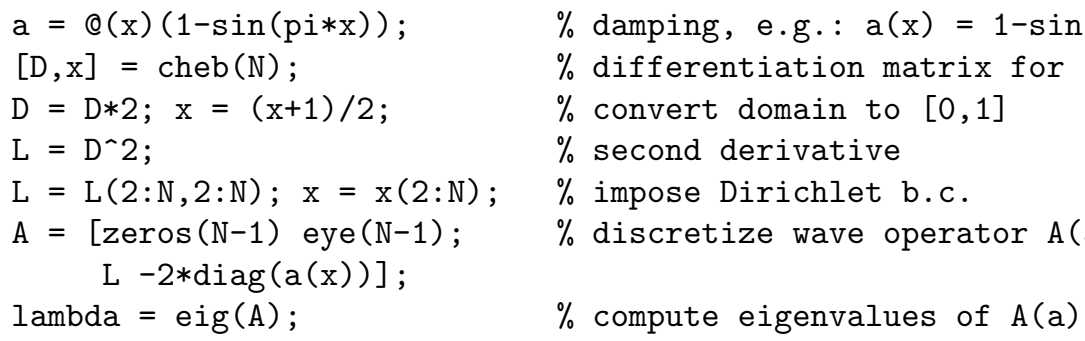

The discretized wave operator $\mathrm{A}$ is of order $2 \mathrm{~N}-2$. In our experience, this high-order discretization delivers roughly $\mathrm{N}$ computed eigenvalues (those nearest the real axis) that appear valid to plotting accuracy, provided the damping function is smooth. Table 1 illustrates the accuracy of the approximation (13) suggested by Theorem 5 for the even damping $a(x)=1-\sin (11 \pi x) \exp \left(-10(x-1 / 2)^{2}\right)$. (The 'exact' eigenvalues were computed using a spectral discretization with $\mathrm{N}=200$, confirmed with results identical to the given precision for $\mathrm{N}=400$. The integrals necessary for $\widetilde{\lambda}_{n}(a)$ were computed using Mathematica and Clenshaw-Curtis quadrature [7].)

Even for $n=1$ the approximation (13) suggested by Theorem 5 is quite good, despite the rather irregular nature of the low-frequency eigenvalues. Such results are typical of the majority of our experiments. Further evidence is provided in Figure 1, which compares the true and approximate eigenvalues for the damping used in Table 1 and two other even functions; all eigenvalues that satisfy $\operatorname{Im} \lambda \in[0,50]$ are included in these plots. (Again, the discretization uses $\mathrm{N}=200$.) An example for which this expansion is inaccurate at low frequencies is described in Section 5.3. 

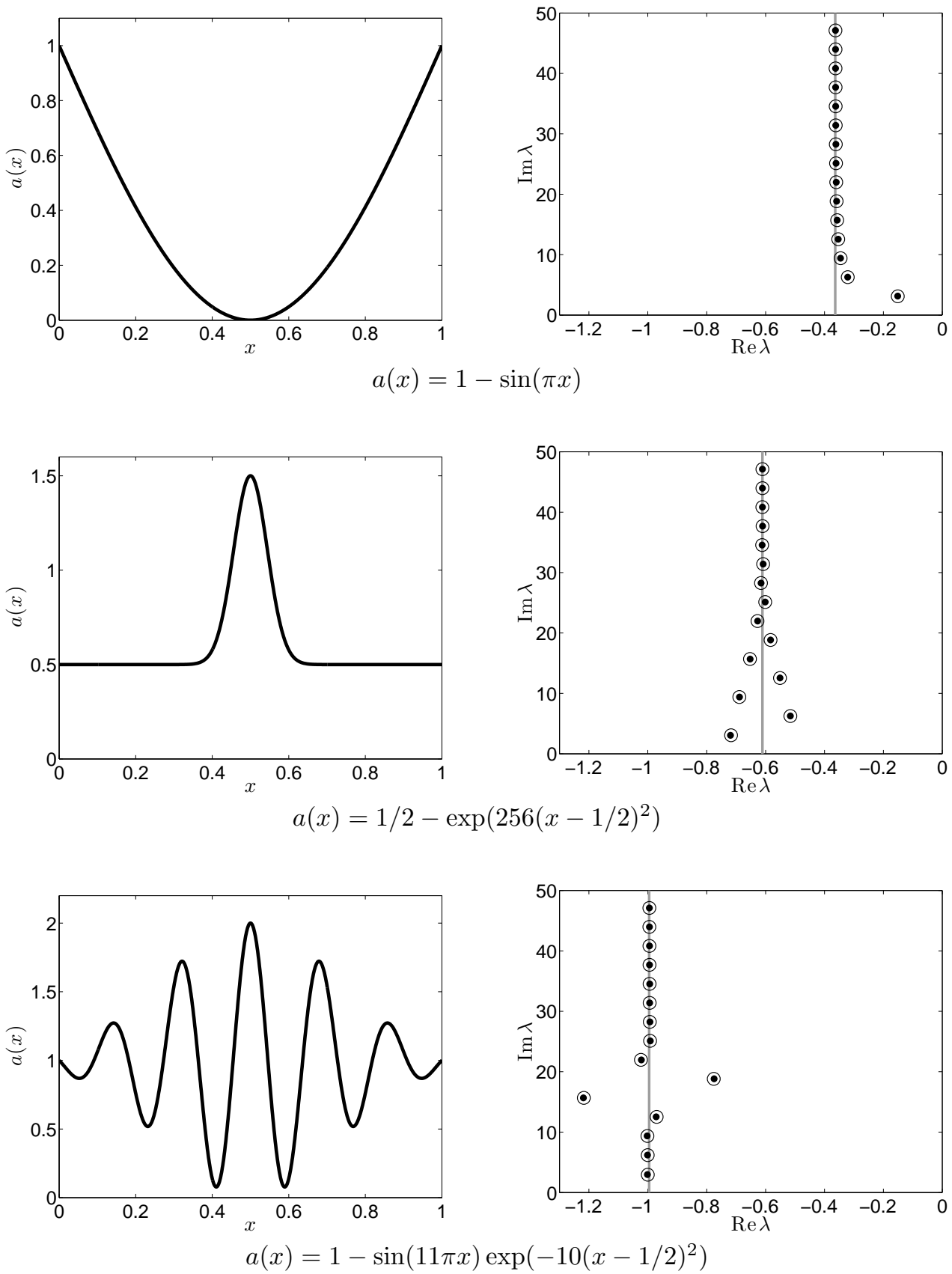

Figure 1. Gallery of even damping functions (left) and corresponding eigenvalues (right). The eigenvalues are shown as dots, with circles showing the approximations $\widetilde{\lambda}_{n}(a)$ given by (13); gray vertical lines mark the asymptote $-a_{0}$. 


\section{The Inverse Spectral Problem}

In the spirit of Lowe, Pilant, and Rundell [8] we argue that each eigenvalue encodes a Fourier cosine coefficient of the desired even damping function. We apply Theorem 5 to reconstruct a damping from a single spectrum. In particular, if $a$ is even (about the midpoint $x=1 / 2)$, then $\widetilde{a}$ is even and $\xi$ is odd, so

$$
\phi(x) \equiv \widetilde{a}(x) \frac{\sinh (2 \xi(x))}{2 \xi(x)} \quad \text { and } \quad \psi(x) \equiv \widetilde{a}(x) \frac{\cosh (2 \xi(x))-1}{2 \xi(x)}
$$

are even and odd, respectively. The expansion in Theorem 5 thus includes in the formula for $\lambda_{n}(a)$ the $n$th Fourier coefficients for $\phi$ and $\psi$,

$$
\int_{0}^{1} \widetilde{a}(x) \frac{\sinh (2 \xi(x))}{2 \xi(x)} \cos (2 n \pi x) \mathrm{d} x, \quad \int_{0}^{1} \widetilde{a}(x) \frac{\cosh (2 \xi(x))-1}{2 \xi(x)} \sin (2 n \pi x) \mathrm{d} x .
$$

It follows that both $\phi$ and $\psi$ are determined by the spectrum, i.e., by

$\phi(x)=\phi_{0}+2 \sum_{n=1}^{\infty}\left(a_{0}+\operatorname{Re}\left(\lambda_{n}\right)+O\left(1 / n^{2}\right)\right) \cos (2 n \pi x)$

and

$\psi(x)=2 \sum_{n=1}^{\infty}\left(\operatorname{Im}\left(\lambda_{n}\right)-n \pi+\frac{1}{2 n \pi} \int_{0}^{1} a^{2}(x) \mathrm{d} x+O\left(1 / n^{2}\right)\right) \sin (2 n \pi x)$,

where

$$
\begin{aligned}
\phi_{0} & =\int_{0}^{1} \widetilde{a}(x) \frac{\sinh (2 \xi(x))}{2 \xi(x)} \mathrm{d} x \\
& =\int_{0}^{1}\left(\int_{0}^{\xi(x)} \frac{\sinh (2 y)}{2 y} \mathrm{~d} y\right)^{\prime} \mathrm{d} x, \quad \text { as } \widetilde{a}=\xi^{\prime}, \\
& =\int_{0}^{\xi(1)} \frac{\sinh (2 y)}{2 y} \mathrm{~d} y-\int_{0}^{\xi(0)} \frac{\sinh (2 y)}{2 y} \mathrm{~d} y=0,
\end{aligned}
$$

as $\xi(0)=\xi(1)=0$. Simple manipulation of (14) reveals

$$
\frac{2 \psi \phi}{\phi^{2}+\psi^{2}}=\tanh (2 \xi)
$$

which uniquely determines $\xi$, and hence the even function $a=a_{0}+\widetilde{a}=a_{0}+\xi^{\prime}$. (Corollary 1 ensures this is the unique even damping with the specified eigenvalues.)

Given knowledge of the spectrum (as obtained from measurements of an unknown string, or specified by a string designer), can we neglect the $O\left(1 / n^{2}\right)$ terms in (15) and (16) to obtain estimates for $\phi$ and $\psi$, and hence $\xi$ and $a$ via (17)? Does this recovered $a$ indeed capture the desired spectrum? We investigate this question for the function $a(x)=1-\sin (\pi x)$ in Figure 2. The top plots show $\widetilde{a}(x)=2 / \pi-\sin (\pi x)$ and $\xi(x)=(\cos (\pi x)+2 x-1) / \pi$; the bottom plots compare $\phi$ and $\psi$ to reconstructions using (15) and (16), truncating the sum at $n=10$. (Eigenvalues for this damping function are illustrated in Figure 1.) We see that $\phi$ is recovered well, but the approximation to $\psi$, while of the correct order of magnitude, is quite inaccurate.

The explanation for this fact lies in the magnitude of the $\xi$ function. Note that $\xi(0)=\xi(1)=0$, and when $a$ is even, we further have that $\xi(1 / 2)=0$ (recall $\xi$ is odd). Coarse estimates follow readily: $\|\xi\|_{L^{\infty}} \leq \frac{1}{4}\|\widetilde{a}\|_{L^{\infty}}$, and, for differentiable $a$,

$$
\|\xi\|_{L^{\infty}} \leq \frac{\|\widetilde{a}\|_{L^{\infty}}}{4}-\frac{\|\widetilde{a}\|_{L^{\infty}}^{2}}{2\left\|a^{\prime}\right\|_{L^{\infty}}} .
$$



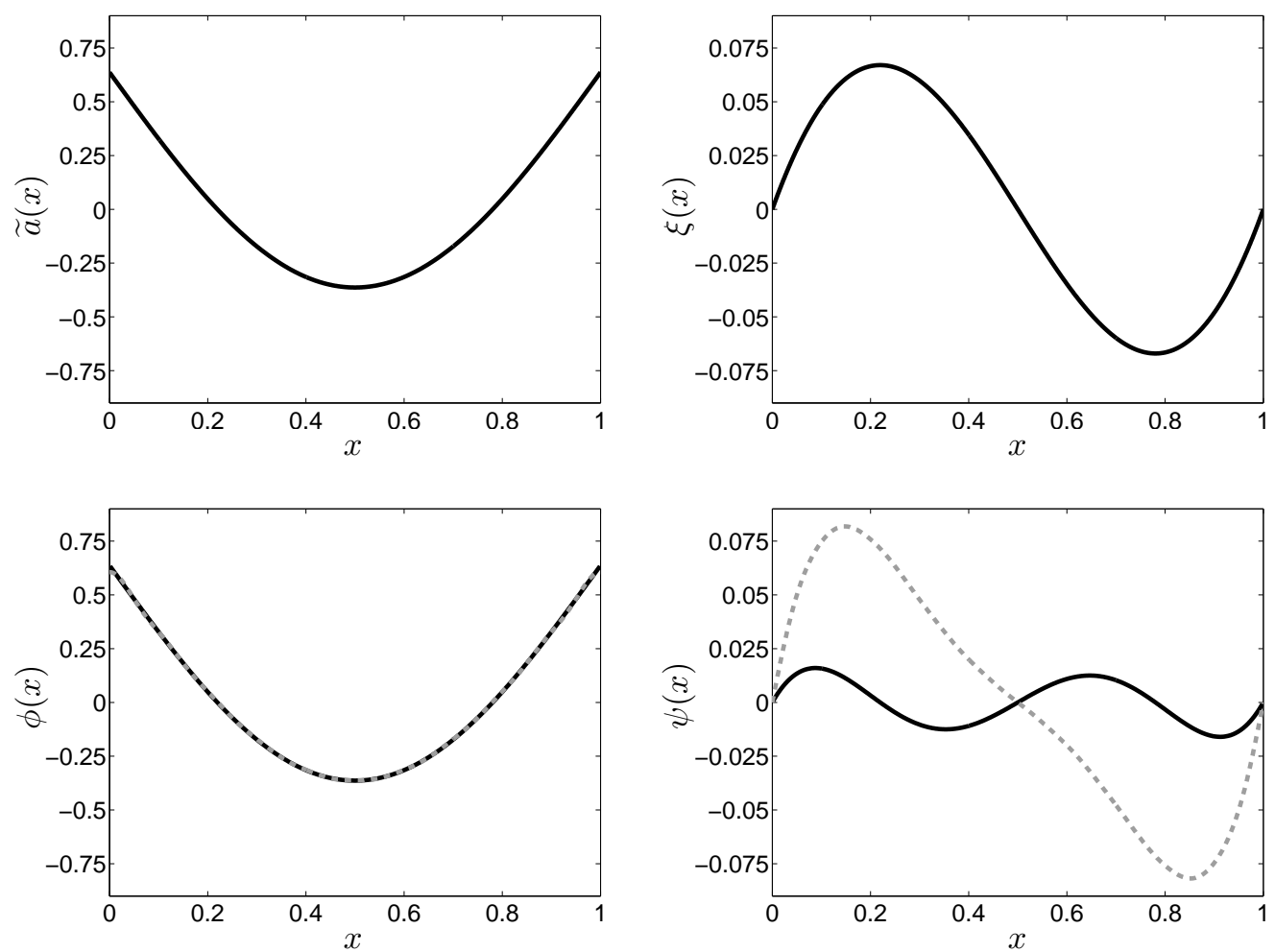

Figure 2. For the example $a(x)=1-\sin (\pi x)$, the top plots show $\widetilde{a}(x)=a(x)-a_{0}$ and $\xi(x)=\int_{0}^{x} \widetilde{a}(s) \mathrm{d} s$. The bottom plots show $\phi$ and $\psi$ (solid black lines) along with the approximations constructed from 10 eigenvalues (dashed gray lines). The two curves in the bottom-left plot are nearly coincident.

Thus, $\xi$ tends to be considerably smaller than the deviation from the mean, $\widetilde{a}$. For small $\xi$ we have the approximations

$\phi(x)=\widetilde{a}(x)\left(1+\frac{2 \xi^{2}(x)}{3}+O\left(|\xi(x)|^{4}\right)\right), \quad \psi(x)=\widetilde{a}(x)\left(\xi(x)+\frac{\xi^{3}(x)}{3}+O\left(|\xi(x)|^{5}\right)\right)$,

thus justifying the approximations

$$
\begin{aligned}
& \phi(x)=\widetilde{a}(x)+O\left(\|\widetilde{a}\|_{L^{\infty}}\|\xi\|_{L^{\infty}}\right) \approx \widetilde{a}(x) \\
& \psi(x)=O\left(\|\widetilde{a}\|_{L^{\infty}}\|\xi\|_{L^{\infty}}\right) \approx 0 .
\end{aligned}
$$

Given a finite set of eigenvalues $\lambda_{1}, \ldots, \lambda_{m}$, we then approximate (cf. [8])

$$
a(x) \approx a_{0}+2 \sum_{j=1}^{m}\left(a_{0}+\operatorname{Re}\left(\lambda_{n}\right)\right) \cos (2 n \pi x) .
$$

\section{Computational Illustrations}

How effective is the recovery scheme (18)? We generate synthetic data using the discretization described at the end of Section 3, then conduct three experiments. 


\subsection{Experiment 1: Recovery of even damping}

For these first experiments, we recover the even damping functions

$$
a(x)=1-\sin (\pi x),
$$

and

$$
a(x)=1-\sin (11 \pi x) \exp \left(-10(x-1 / 2)^{2}\right)
$$

using $m=5,10$, and 40 eigenvalues. The eigenvalues are 'exact', but the value of $a_{0}$ is estimated using the $m$ th eigenvalue,

$$
a_{0} \approx-\operatorname{Re} \lambda_{m}
$$

as might be done in a physical experiment. The very satisfactory reconstructions from (18) are shown in Figures 3 and 4. (A more accurate estimate of $a_{0}$ could likely be obtained through Richardson extrapolation; given the noise that affects practical measurements, one might instead approximate $a_{0}$ by averaging the real parts of the measured eigenvalues of highest frequency.)

\subsection{Experiment 2: Recovery of even damping from noisy data}

Our next experiment works again with $a(x)=1-\sin (11 \pi x) \exp \left(-10(x-1 / 2)^{2}\right)$, but now in the presence of noise. In particular, we take $m=20$ 'exact' eigenvalues and perturb their real and imaginary parts by normally distributed noise (mean zero, standard deviation 0.01). Figure 5 shows the recovery of a single damping function, as well as the mean of the functions obtained from twenty trials. (For each trial we approximate $a_{0}$ with $-\operatorname{Re} \lambda_{m}$.)

\subsection{Experiment 3: Attempted recovery of extreme damping}

Can we achieve arbitrarily rapid energy decay in a string with even damping? For bounded variation $a$, Cox and Zuazua [3] equate the asymptotic rate of energy decay with the spectral abscissa of $A(a)$ (the largest real part of any point in the spectrum). Hence to maximize energy decay, one seeks damping functions $a$ for which the spectrum falls far to the left of the imaginary axis. Among constant dampings, $a(x) \equiv \pi$ is optimal, giving a spectral abscissa of $-\pi$. It had been conjectured that the best asymptotic decay rate was achieved by this constant damping [9]; however, Castro and Cox [10] showed that the family of (uneven) damping functions

$$
a(x)=\frac{1}{x+1 / k}
$$

produces arbitrarily strong asymptotic damping as $k \rightarrow \infty$, i.e., the spectral abscissa can be made arbitrarily negative by taking $k$ sufficiently large. Is such extreme spectral behavior possible for an even damping function? To investigate, we discretize the operator with $a(x)=(x+1 / k)^{-1}$ for various $k$, then use (18) to recover an even damping coefficient, hopefully with the same spectrum.

Our results (with recovery of the even damping function based on 50 'exact' eigenvalues from the uneven damping function $\left.a(x)=(x+1 / k)^{-1}\right)$ are shown in

Figure 6. One observes that for small values of $k$, our procedure constructs an even damping that is approximately as effective as the uneven function $a(x)=(x+1 / k)^{-1}$. However, when $k$ is sufficiently large that the spectral abscissa is smaller than $-\pi$, the even damping spawns a pair of real eigenvalues that correspond to an overdamped 

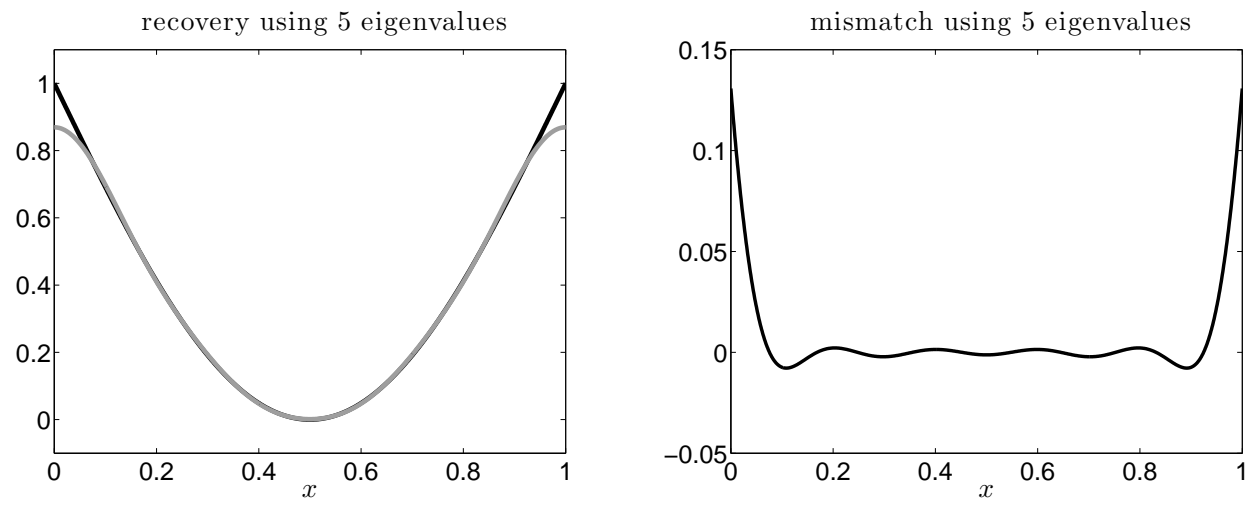

recovery using 10 eigenvalues

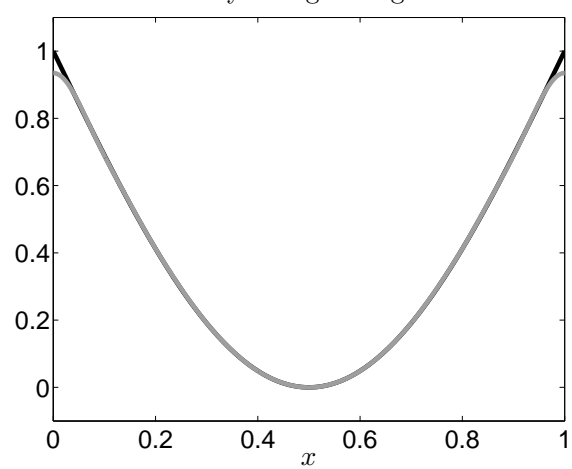

mismatch using 10 eigenvalues

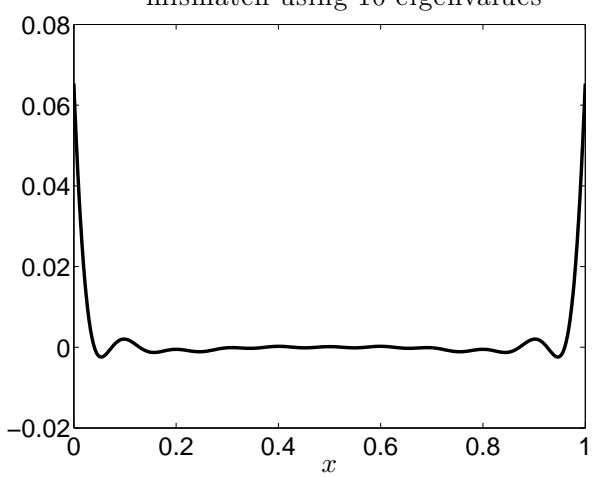

recovery using 40 eigenvalues
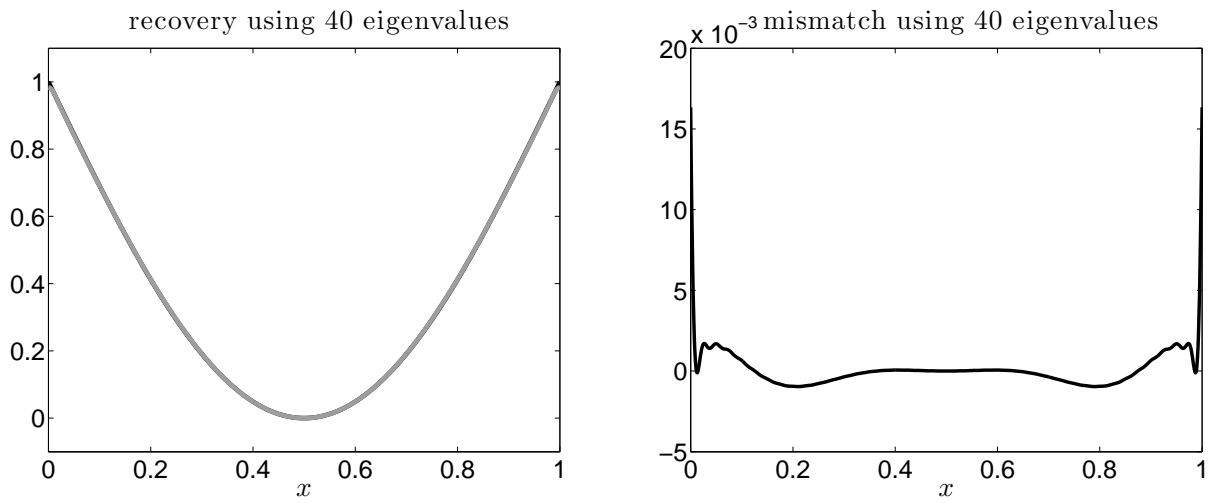

Figure 3. Recovery of even damping $a(x)=1-\sin (x)$ using 5,10 , and 40 eigenvalues. In the left plot, the exact $a$ (black) is mostly obscured by the the recovered $a$ (gray). 

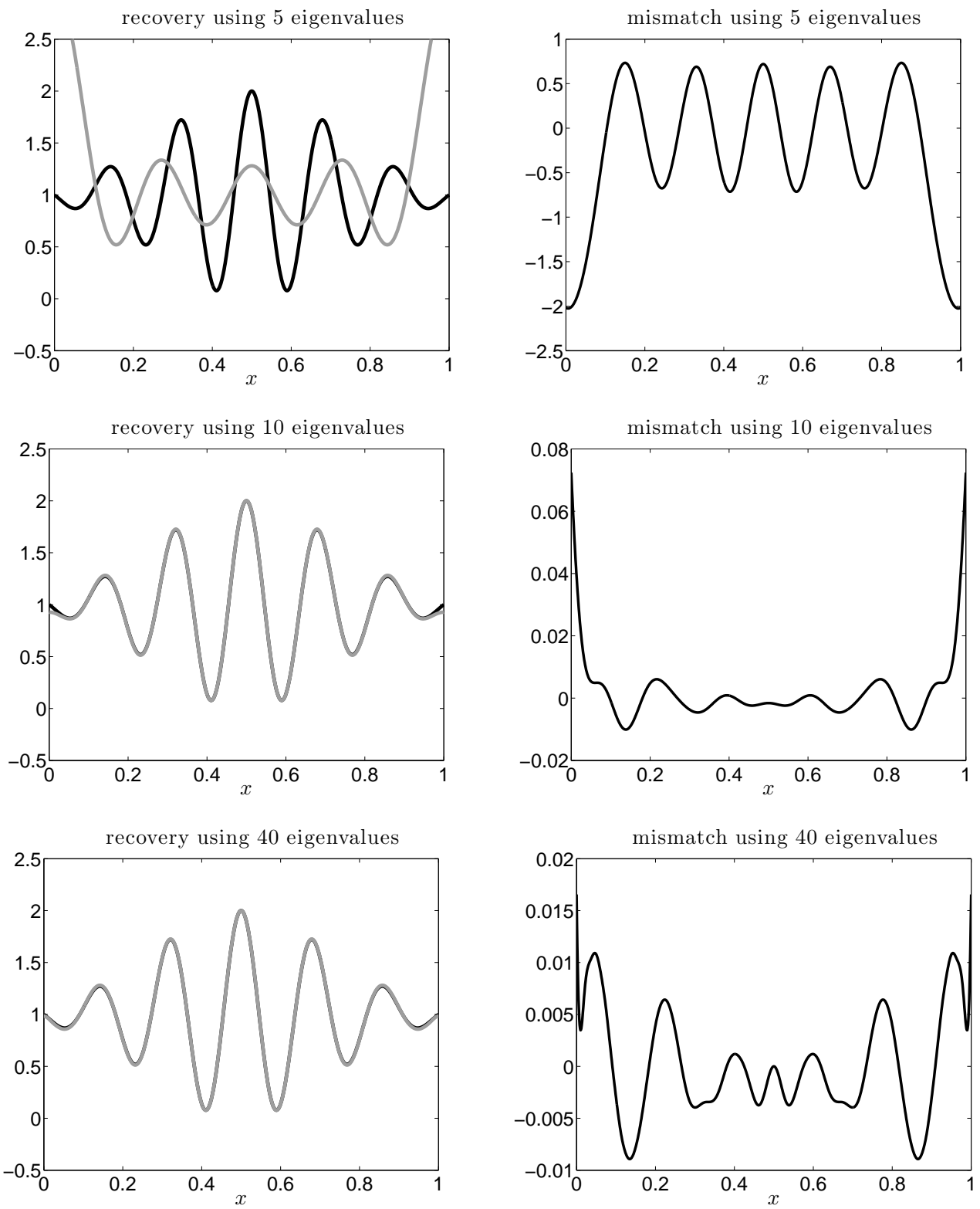

Figure 4. Recovery of even damping $a(x)=1-\sin (11 \pi x) \exp \left(-10(x-1 / 2)^{2}\right)$ using 5, 10, and 40 eigenvalues. In the left plot, the exact $a$ (black) is mostly obscured by the the recovered $a$ (gray) for $m=10$ and $m=40$. 

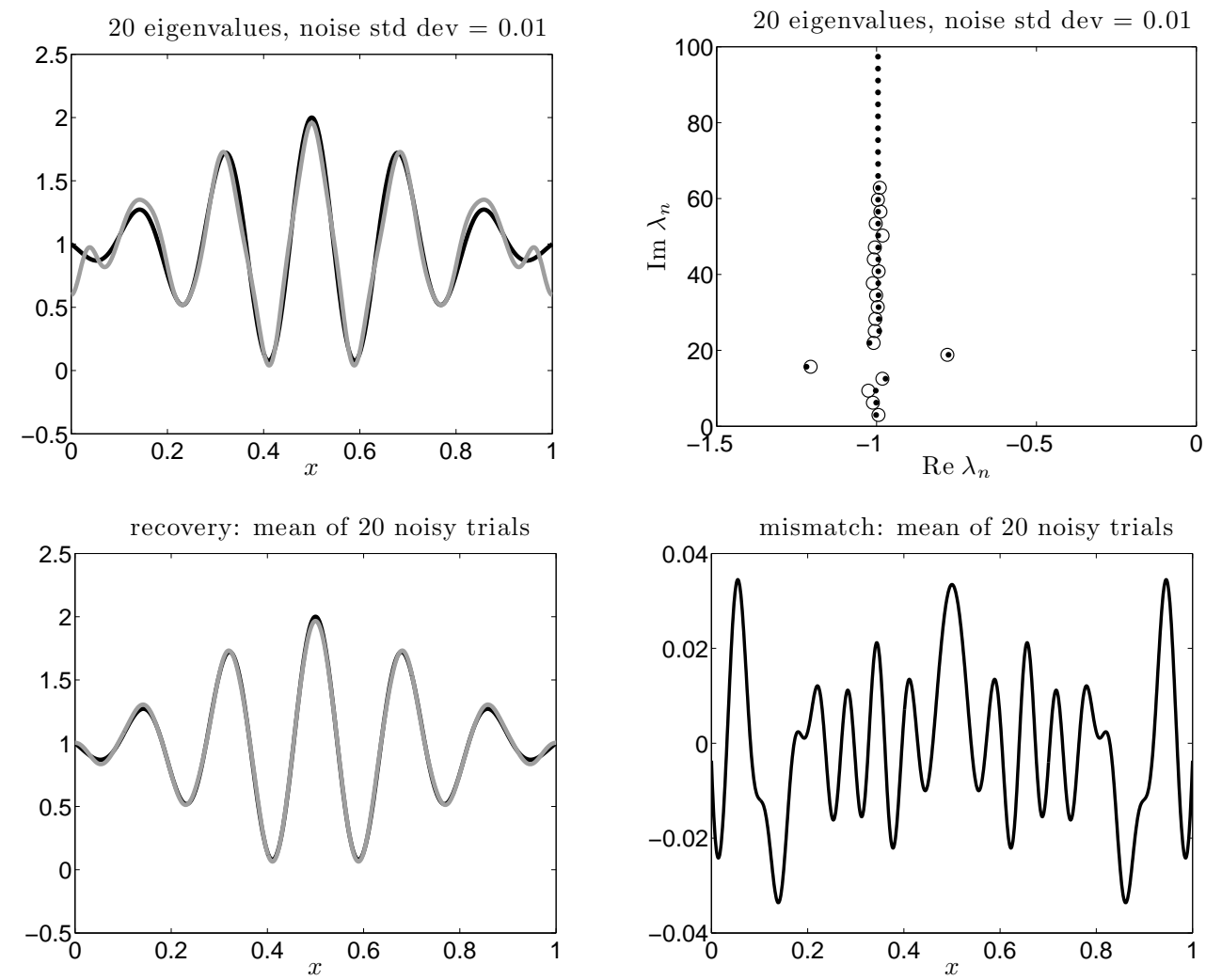

Figure 5. Recovery of damping $a(x)=1-\sin (11 \pi x) \exp \left(-10(x-1 / 2)^{2}\right)$ using 20 eigenvalues, with noise. Real and imaginary parts of the true eigenvalues are each perturbed by normally distributed noise of zero mean and standard deviation 0.01. The plots show one recovered damping function (top left) and the twenty perturbed eigenvalues that produced it (top right, with true eigenvalues as dots and perturbed data as circles), along with the mean damping function from twenty such trials (bottom left) and the associated mismatch with the true damping function (bottom right).

system, and the spectral abscissa increases. While this procedure thus fails to produce an even damping with faster asymptotic decay than the best constant, an example of Freitas [11] (example i on p. 394, scaled for our domain)

$$
a(x)=\pi\left(\frac{3.1133}{2}+1.4896 \cos (2 \pi x)\right)
$$

is apparently more potent. Figure 7 illustrates that this damping has large amplitude, as reflected in the magnitude of the coefficients in (13) and other relevant quantities:

$a_{0}=1.55665 \pi, \int_{0}^{1} a^{2}(x) \mathrm{d} x=3.5326133025 \pi^{2},\|\widetilde{a}\|_{L^{\infty}}=1.4896 \pi,\|\xi\|_{L^{\infty}}=0.7448$.

Given these magnitudes, it is no surprise that the approximation (13) is inaccurate at low frequencies, as is the recovery from (18) shown in Figure 7 . The lowest frequency eigenvalues for this particular damping are especially sensitive to perturbations: Figure 8 compares $\varepsilon$-pseudospectra [12] of $A(a)$ for the damping (20) to Freitas's example (21) (computed using a discretization of the appropriate energy inner 

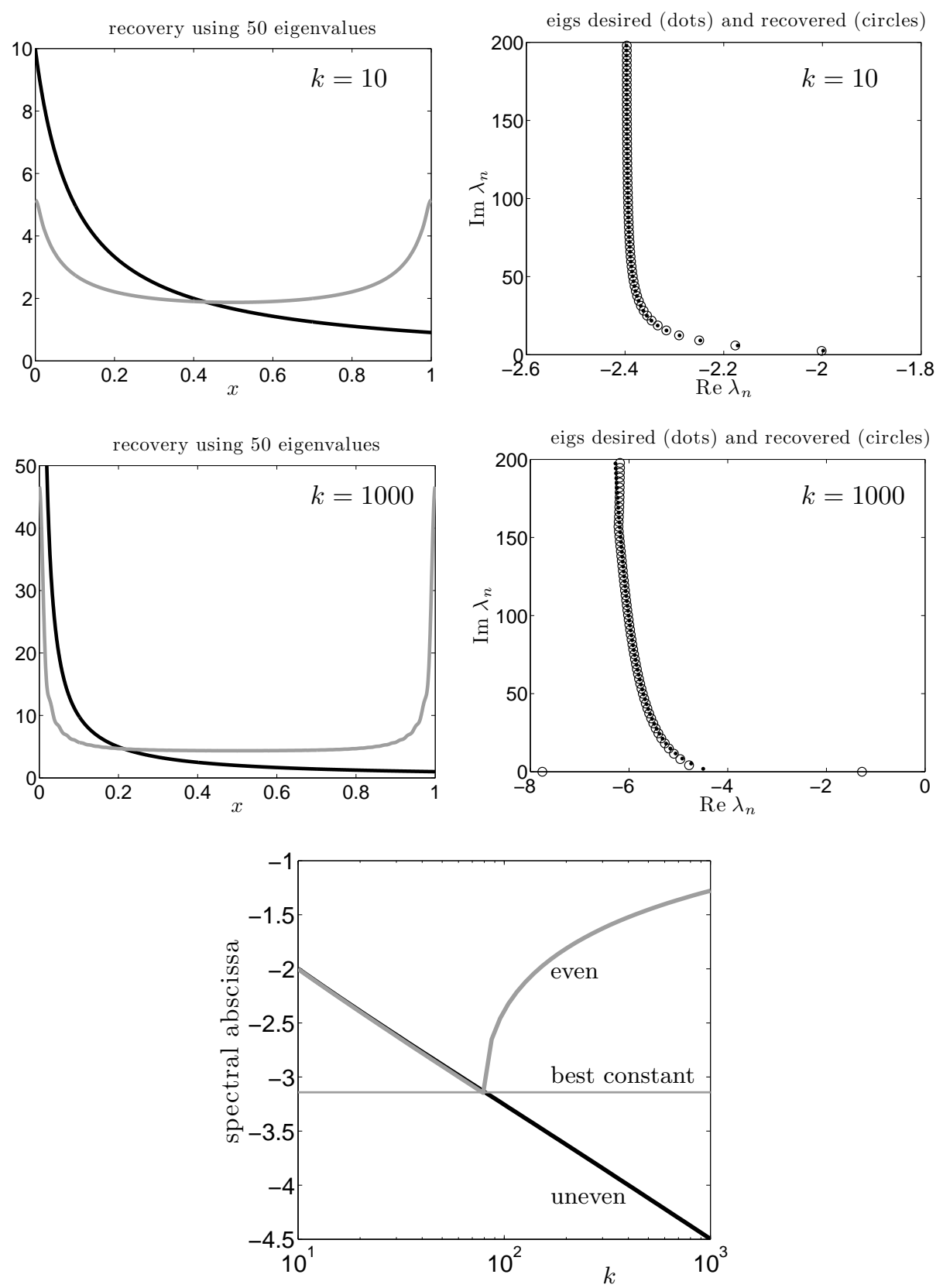

Figure 6. Attempt to recover an even damping as effective as $a(x)=(x+1 / k)^{-1}$ for large $k$. The plots on the left show the uneven damping function (black line) and the recovered even function (gray line). The plots on the right show the eigenvalues for the uneven damping (dots) and recovered even damping (circles). For $k=10$ (top row), the even function is as effective as the uneven one; for $k=1000$ (middle row), the even damping leads to a pair of real eigenvalues, and overdamping. The bottom plot shows that the even construction breaks down when $k$ is sufficiently large that the spectral abscissa for $a(x)=(x+1 / k)^{-1}$ is smaller than that for the optimal constant, $-\pi$, marked by the horizontal line. 

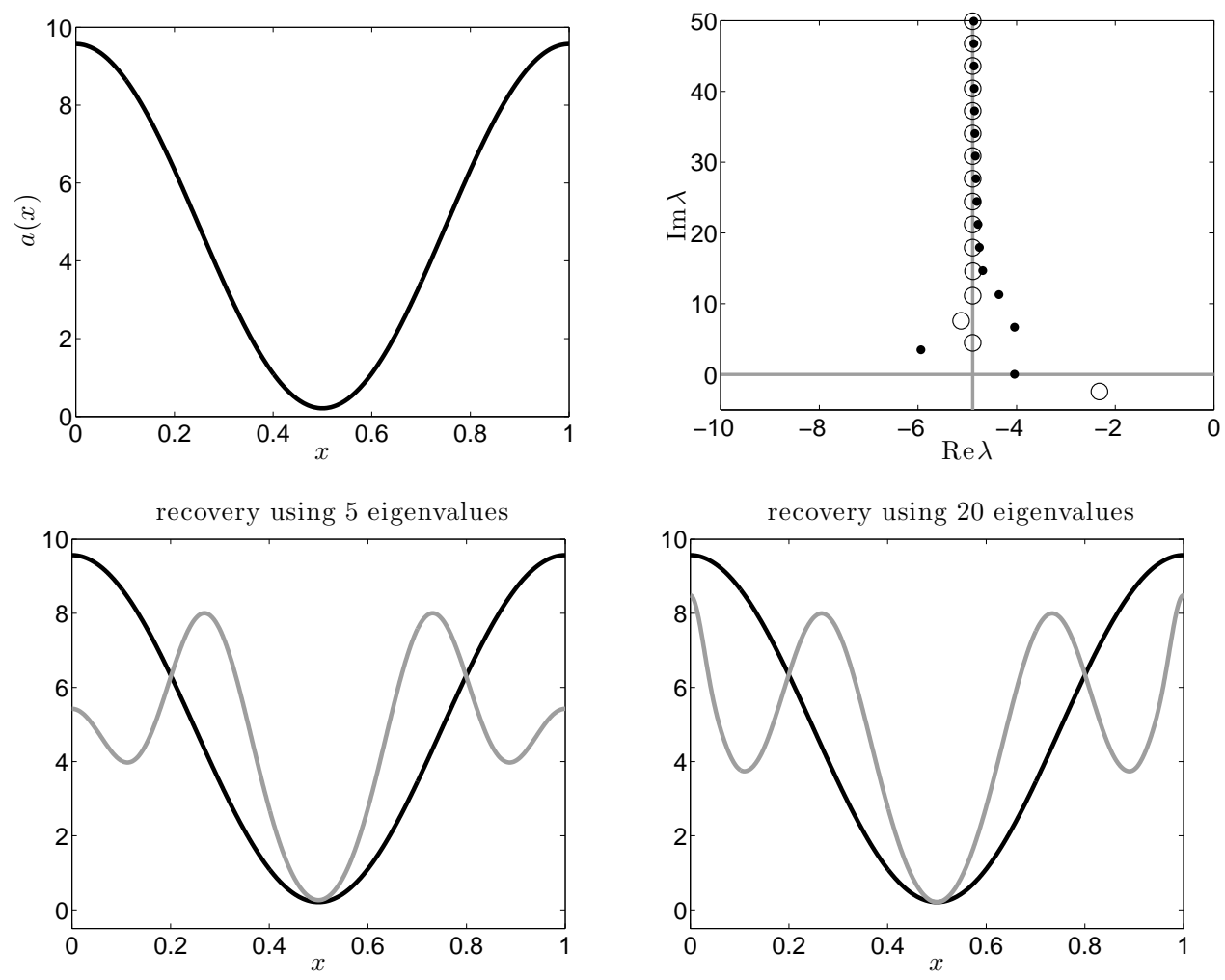

Figure 7. An example of potent even damping (21) from Freitas [11]. Upper left: the damping, $a$. Upper right: The eigenvalues of $A(a)$ are shown as dots, with circles showing the approximations $\widetilde{\lambda}_{n}(a)$ given by (13); the gray vertical line marks the asymptote $-a_{0}$. The rightmost eigenvalue is very near the real axis $\left(\lambda_{1}(a) \approx-4.0428+0.0343 i\right)$, while its approximation $\widetilde{\lambda}_{1}(a)$ is in the lower half-plane. Lower left and right: attempted reconstruction of $a$ using the first 5 and 20 eigenvalues.

product). For $\varepsilon=10^{0}$ (outermost contours), the $\varepsilon$-pseudospectrum for damping (20) comprise disks centered at each eigenvalue with radius only slightly larger than $\varepsilon$ : a perturbation to $A(a)$ of norm 1 can only move eigenvalues within these boundaries. For Freitas's example, the corresponding pseudospectrum contains points much farther from the spectrum, reflecting higher sensitivity to perturbations. This fact may shed light on the poor accuracy of the approximation (13) at low frequencies, and the consequent error in the recovered damping.

\section{Acknowledgements}

We thank Jeffrey Hokanson for his helpful comments, and gratefully acknowledge the support of NSF grants DMS-0505893 and DMS-CAREER-0449973. The numerical computations were facilitated by MATLAB (with L. N. Trefethen's tools for spectral methods [7], Marcel Leutenegger's erfz command, and T. G. Wright's EigTool software [13]) and Mathematica. 

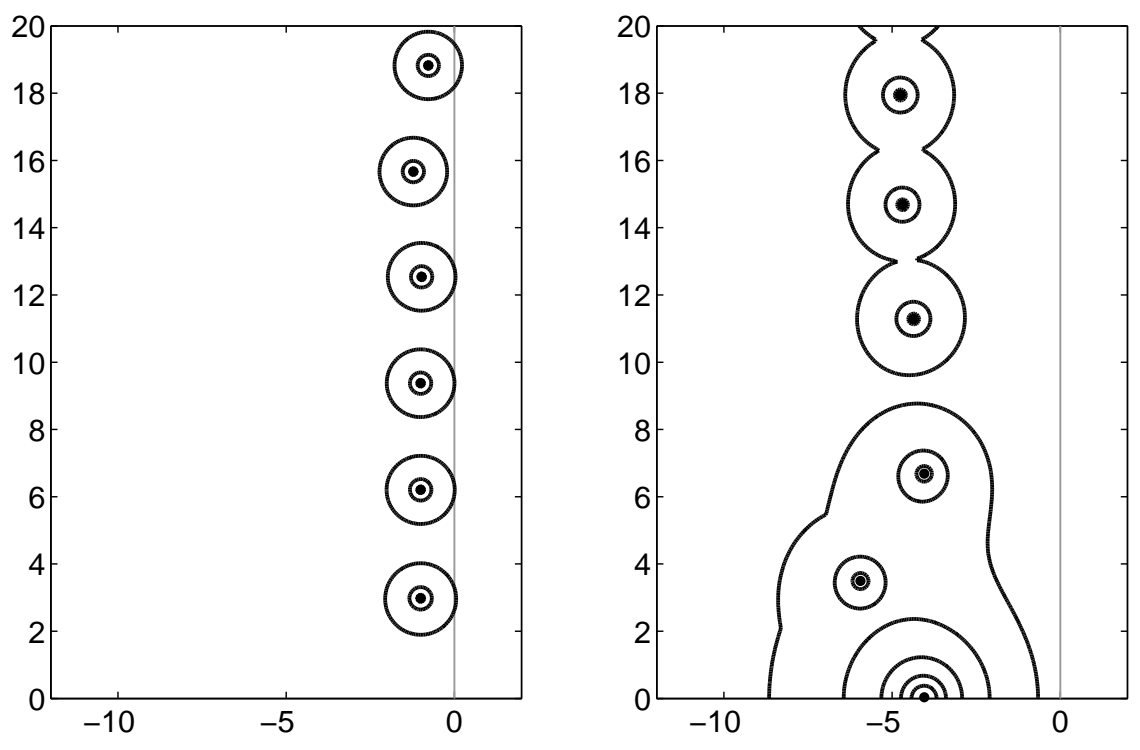

Figure 8. Spectra and $\varepsilon$-pseudospectra of the damping (20) (left) and Freitas's example (21) (right) for $\varepsilon=10^{0}, 10^{-.5}, 10^{-1}, 10^{-1.5}, 10^{-2}$. The gray vertical lines mark the imaginary axis.

\section{References}

[1] Masahiro Yamamoto. Inverse spectral problem for systems of ordinary differential equations of first order. I. J. Fac. Sci. Univ. Tokyo, 35:519-546, 1988.

[2] S. J. Cox and R. Knobel. An inverse spectral problem for a first order system of ordinary differential equations. Integral Equations Operator Theory, 25:147-162, 1996.

[3] Steven Cox and Enrique Zuazua. The rate at which energy decays in a damped string. Comm. Partial Diff. Eqns., 19:213-243, 1994.

[4] F. R. Gantmacher and M. G. Krein. Oscillation Matrices and Kernels and Small Vibrations of Mechanical Systems. AMS Chelsea, Providence, RI, revised edition, 2002.

[5] Jürgen Pöschel and Eugene Trubowitz. Inverse Spectral Theory. Academic Press, Boston, 1987.

[6] Denis Borisov and Pedro Freitas. Eigenvalue asymptotics, inverse problems and a trace formula for the linear damped wave equation. J. Diff. Eq., 247:3028-3039, 2009.

[7] Lloyd N. Trefethen. Spectral Methods in MATLAB. SIAM, Philadelphia, 2000.

[8] Bruce D. Lowe, Michael Pilant, and William Rundell. The recovery of potentials from finite spectral data. SIAM J. Math. Anal., 23:482-504, 1992.

[9] Steven J. Cox and Michael L. Overton. Perturbing the critically damped wave equation. SIAM J. Appl. Math., 56:1353-1362, 1996.

[10] Carlos Castro and Steven J. Cox. Achieving arbitrarily large decay in the damped wave equation. SIAM J. Control Optim., 39:1748-1755, 2001.

[11] Pedro Freitas. Optimizing the rate of decay of solutions of the wave equation using genetic algorithms: a counterexample to the constant damping conjecture. SIAM J. Control Optim., $37: 376-387,1998$

[12] Lloyd N. Trefethen and Mark Embree. Spectra and Pseudospectra: The Behavior of Nonnormal Matrices and Operators. Princeton University Press, Princeton, NJ, 2005.

[13] Thomas G. Wright. EigTool, 2002. Software available at http://www.comlab.ox.ac.uk/projects/pseudospectra/eigtool. 